\title{
Risking Everything in Obsessive-Compulsive Disorder: An Analogue Decision-Making Study
}

\author{
James Croft $^{1}$ · Jessica R. Grisham ${ }^{1} \cdot$ Andrew Perfors $^{2} \cdot$ Brett K. Hayes $^{1}$ \\ Accepted: 7 June 2021 / Published online: 22 November 2021 \\ (c) The Author(s), under exclusive licence to Springer Science+Business Media, LLC, part of Springer Nature 2021
}

\begin{abstract}
An experiment examined decision-making processes among nonclinical participants with low or high levels of OCD symptomatology $(\mathrm{N}=303)$. To better simulate the decision environments that are most likely to be problematic for clients with OCD, we employed decision tasks that incorporated "black swan" options that have a very low probability but involve substantial loss. When faced with a choice between a safer option that involved no risk of loss or a riskier alternative with a very low probability of substantial loss, most participants chose the safer option regardless of OCD symptom level. However, when faced with choices between options that had similar expected values to the previous choices, but where each option had some low risk of a substantial loss, there was a significant shift towards riskier decisions. These effects were stronger when the task involved a contamination based, health-relevant decision task as compared to one with financial outcomes. The results suggest that both low and high symptom OC participants approach decisions involving risk-free options and decisions involving risky alternatives in qualitatively different ways. There was some evidence that measures of impulsivity were better predictors of the shift to risky decision making than OCD symptomatology.
\end{abstract}

Keywords Obsessive-compulsive disorder $\cdot$ Black swan $\cdot$ Decision-making $\cdot$ Risk-taking $\cdot$ Impulsivity

\section{Introduction}

OCD is a disorder characterized by the presence of obsessions and/or compulsions (American Psychiatric Association [APA], 2013). Obsessions are recurrent and persistent thoughts, urges, or images, experienced as intrusive and unwanted, that cause marked anxiety. Compulsions are repetitive behaviors or mental acts that an individual engages in to neutralize obsessions. In many ways, individuals with OCD present as risk-averse and harm-avoidant (Cicolini \& Rees, 2003). Indeed, compulsions are conceptualised as efforts to reduce perceived threat and anxiety even when the objective threat of risk is low (APA, 2013). Hence, a person with OCD may decide that they need to wash their hands repeatedly even though there is a negligible risk that they have been contaminated or decide that additional checking

James Croft

james.croft@unswalumni.com

1 School of Psychology, University of New South Wales, Mathews Building, Kensington, Sydney, NSW 2052, Australia

2 School of Psychological Sciences, University of Melbourne, Melbourne, Australia of power points is needed even though there is a very small risk of an electrical fire.

There is substantial evidence that the most effective treatment approach for OCD is exposure and response prevention (ERP; Olatunji et al., 2013). ERP is a cognitive-behavioural treatment that involves repeated exposure to situations that clients with OCD perceive as excessively risky (e.g., public bathrooms), without performing compulsions (e.g., handwashing). Thus, ERP provides opportunities for the individual to tolerate minimal levels of risk and learn that these situations are generally safe, thus extinguishing obsessional fears (Koran \& Simpson, 2013). Despite its efficacy, a significant portion of individuals with OCD do not adhere to ERP homework assignments (Abramowitz et al., 2002) or decline ERP treatment, often due to extreme anxiety about engaging in "risky" exposures. Understanding how individuals with OCD perceive and interpret risk - and how these perceptions influence the decisions that they make - is potentially crucial to targeting risk perception in OCD, thereby facilitating uptake and engagement in effective cognitive-behavioural approaches.

Given the risk-averse clinical presentation of clients with OCD and its impact on treatment outcomes, a considerable body of research has thus examined how those 
with OCD make decisions involving risk (see Aranovich et al., 2017 for a review). In such studies, those with OCD are compared with healthy controls on tasks where they are offered a choice between a safer option (e.g., a high probability of winning a small reward) and a riskier option (e.g., where there is some probability of winning a larger reward, but also some risk of losing it). Such studies, however, have yet to establish a clear and consistent relationship between OCD symptoms and risky decision-making. On the one hand, a number of studies have suggested that those with OCD show greater risk aversion (i.e., are more likely to avoid decision options that involve risk) than controls (Admon et al., 2012; Foa et al., 2002; Sip et al., 2016). Other studies, however, have found no differences in the risky decisions of people with OCD and controls (Pushkarskaya, et al., 2015, 2017; Starcke et al., 2010), or have found lower levels of risk aversion (i.e., risk-seeking) in those with OCD (Aranovich, et al., 2017; Grassi, et al., 2015).

In part, this inconsistency in results may reflect the use of a variety of decision tasks across different studies that vary in the size and probability of gains and losses. It may also reflect the use of tasks that are not ideally suited for probing the types of decisions that are most problematic for those with OCD. As illustrated in the earlier examples, such problems frequently arise when those with OCD are faced with outcomes that have low objective probability (e.g., contamination after hand washing; overlooking a live power switch) but very serious negative consequences (e.g., developing or transmitting an illness; a house fire). Events that confer an extremely low probability of catastrophic loss are referred to in the decision-making literature as 'black swans' (Hertwig \& Erev, 2009; Taleb, 2007). However, despite their clinical relevance, no studies of decision-making in OCD have employed tasks with a black swan structure. In previous studies of decisionmaking in OCD, the probability of negative outcomes is usually no smaller than $5 \%$, and the losses are far from catastrophic (usually just a small portion of one's points or money). Pushkarskaya et al. (2015), for example, used outcome probabilities varying from 0.13 to 0.75 with possible monetary gains and losses between $\$ 5$ and $\$ 125$.

This example illustrates a second limitation of previous studies of decision-making in OCD: decision outcomes are typically financial. This contrasts with the key concerns in clinical obsessions, which frequently involve negative outcomes involving personal responsibility for the health or safety of oneself or loved ones (Salkovskis, et al., 2000; Schulze et al., 2018). In the absence of decision tasks that simulate such consequences, it remains unclear whether the results from previous decision-making studies can be generalised to decision-making in OCD.
The main aim of the current work, therefore, was to examine decision-making in OCD using tasks that better simulate the decision environments that are most likely to be problematic for these clients. To this end we employed a decision task that incorporated black swan choice options, which was administered to non-clinical samples with varying levels of OCD symptomatology. Our task included decision options with a very low probability (e.g., 0.001) of a serious negative consequence (losing all accumulated rewards). Notably, alternate versions of the task implemented different types of decision outcomes: either financial outcomes, as per previous work in this area, or personally relevant health outcomes (avoiding a deadly virus).

Our black-swan tasks were patterned after decision tasks developed by Perfors and Van Dam (2018). In the Perfors and Van Dam (2018) studies, an online sample of healthy adults was provided with a large starting endowment of virtual points (e.g., 7000) and asked to make decisions that would maximise their final points balance, which could then be exchanged for a monetary reward. Participants then carried out one of two different decision tasks. The "one-risk" task involved a choice between two options - the "safer" option offered a moderate probability of small gain and no probability of a loss; the more risky option offered a moderate probability of a larger gain with the simultaneous but very small probability (e.g., 1 in 1000) of losing all accumulated points (a "black swan" outcome). In contrast, in the "two-risk" task, both of the choice options contained a low risk of a catastrophic black swan outcome, although there was a large difference in the relative probability of each outcome (the black swan outcome was 15 times more likely in one option than the other). According to the expected utility theory of decision-making (Buchholz \& Schymura, 2012; von Neumann \& Morgenstern, 1944), when points endowments are large, the safer option is the optimal choice for both one-risk and two-risk tasks (simulations demonstrating this can be found in the Open Science Framework archive for this project).

Interestingly, Perfors and Van Dam (2018) found that choice patterns differed between the one-risk and two-risk tasks. In the one risk task, a majority of participants chose the safer option. However, this pattern reversed for the two-risk task, where a majority chose the riskier option, even though the difference in expected values between the choice options was similar to the options in the one-risk task. Perfors and Van Dam (2018) speculated that their nonclinical participants encoded the choice options in the two versions of the task in qualitatively different ways. On the one-risk task, they may have preferred the safer option because it involved zero risk of a black swan loss. However, when there was a choice between options that both involved some risk, people appeared to ignore the 
differences in relative probability and opted for the riskier option, which offered larger potential rewards. The same shift in risky choice between one-risk and two-risks tasks was found when people made "one shot" choices on each task as well as when they were asked to indicate their choice policy across 2000 repetitions of the tasks.

\section{The Current Study}

To examine the impact of OCD on decision making involving black swan options, we administered both the one-risk and two-risk choice tasks developed by Perfors and van Dam (2018) to participants with either high or low levels of OCD symptoms, as measured by the ObsessiveCompulsive Inventory-Revised (OCI-R; Foa et al., 2002). There is substantial evidence to support the utility and relevance of analogue samples such as this for understanding OCD (Abramowitz et al., 2014). Unlike Perfors and van Dam (2018), all our participants completed both the one-risk and two-risk tasks. This allowed us to directly examine individual stability or shifts in preference for risky options involving black swan outcomes.

For those with low OCD symptoms ("low OC" participants), we expected to replicate the pattern reported by Perfors and van Dam (2018), with a shift towards the riskier options in the two-risk task. The main research question was to examine whether those high in OCD symptoms ("high OC" participants) showed the same shift in risky choice involving black swan outcomes. If so, this would indicate continuity between the decision-making processes in those low and high on OCD symptoms. In contrast, accounts that suggest greater risk aversion in those with OCD (e.g., Admon et al., 2012; Sip, et al., 2016) predict that high OC participants would show an exaggerated shift in risky choice involving black swan outcomes (i.e., an even greater shift towards the riskier options in the tworisk task) relative to low OC participants.

A further important question was the extent to which decision-making patterns in low and high OC participants are similar across different kinds of scenarios (financial vs contamination-related). One possibility suggested by the central role of health-related concerns and personal responsibility in common obsessions (Schulze, et al., 2018) is that decisions involving serious health consequences (e.g., contamination with a potentially deadly virus) will lead to higher levels of risk aversion (e.g., a greater preference for the safer options in the one-risk task), especially among high OC participants. ${ }^{1}$

A secondary goal of the current study was to explore the extent to which patterns of decision-making in black swan

$\overline{1}$ All data was collected in 2019 prior to the COVID-19 pandemic. scenarios are specifically related to OCD symptomatology, as compared with other trans-diagnostic traits such as impulsivity. Previous work indicates that those with OCD often show poorer response inhibition (Abramovitch et al., 2015) and make more impulsive choices (Sohn et al., 2014) than control participants. Although compulsivity and impulsivity were traditionally considered polar ends of the risk-averse/ risk-seeking spectrum, recent models conceptualise them as overlapping constructs that are both linked to problems with top-down inhibitory control (Fontenelle et al., 2011). Thus the behavioural features of OCD may be driven by both compulsivity (repetitive behaviours without adaptive function) and impulsivity (reacting to stimuli in an unplanned fashion with reduced concern for consequences) (Fineberg et al., 2014). This view of impulsivity and compulsivity is consistent with findings of co-existing risk aversion and impulsivity in OCD, such as a preference to avoid risky situations (e.g., lower adjusted mean pumps on Balloon Analogue Risk Task) paired with a difficulty inhibiting urges (e.g., greater future discounting on Delay Discounting Task) and stopping actions once commenced (e.g., poorer response inhibition on Stop Signal Task; Sohn et al., 2014).

To disentangle the effects of OCD symptoms and impulsivity on black swan decision making, the current study also included self-report measures of impulsivity (Barratt Impulsiveness Scale; Stanford et al., 2009) and cognitive reflection (Cognitive Reflection Test; Frederick, 2005). A measure of general psychopathology (Depression, Anxiety, Stress Scales - Brief Version [DASS-21]; Lovibond \& Lovibond, 1995) was also included to examine whether black swan decision making was affected by comorbid clinical symptoms. Additionally, given that all decisions involved some evaluation of quantitative concepts (e.g., outcome probability, value of rewards or losses), we included a test of statistical reasoning ability (Berlin Numeracy Test; Cokely et al., 2012).

\section{Method}

\section{Participants}

An initial pool was recruited via a brief screener task on Amazon Mechanical Turk (MTurk). To be eligible, individuals had to be US residents and have completed at least 1000 prior tasks on MTurk with a $95 \%$ approval rating. A total of 902 individuals completed the screener and were paid US $\$ 0.50$. The screener task consisted of demographic questions followed by the OCI-R (Foa et al., 2002). Those who scored in the top third or bottom third of the OCI-R distribution were invited to participate in the main study. A total of 322 participants accepted the invitation and completed the study, approximately one week after the screen. The OCI-R 
Table 1 Summary of demographics and individual differences data for low OC and high OC participants

\begin{tabular}{|c|c|c|c|c|}
\hline & \multicolumn{2}{|l|}{ Low OC } & \multicolumn{2}{|l|}{ High OC } \\
\hline & $M(S D)$ & Range/Max & $M(S D)$ & Range/Max \\
\hline OCI-R Total Score & $5.78(4.79)$ & $0-20 / 72$ & $39.73(9.92)$ & $22-69 / 72$ \\
\hline Washing & $0.41(0.99)$ & $0-6 / 12$ & $6.42(2.95)$ & $0-12 / 12$ \\
\hline Checking & $0.82(1.38)$ & $0-6 / 12$ & $7.07(2.866)$ & $1-12 / 12$ \\
\hline Ordering & $1.82(2.18)$ & $0-9 / 12$ & $7.86(2.60)$ & $1-12 / 12$ \\
\hline Obsessing & $0.96(1.69)$ & $0-9 / 12$ & $6.91(3.11)$ & $0-12 / 12$ \\
\hline Neutralizing & $0.29(0.83)$ & $0-5 / 12$ & $5.41(3.32)$ & $0-12 / 12$ \\
\hline Hoarding & $1.48(1.69)$ & $0-7 / 12$ & $6.06(3.02)$ & $0-12 / 12$ \\
\hline Age (years)* & $40.25(12.43)$ & $18-77$ & $34.36(11.08)$ & $19-72$ \\
\hline \multicolumn{5}{|l|}{ DASS-21 } \\
\hline Total*** & $10.44(13.01)$ & $0-64 / 126$ & $59.44(27.82)$ & $0-112 / 126$ \\
\hline Depression*** & $3.99(7.87)$ & $0-42 / 42$ & $20.10(11.80)$ & $0-42 / 42$ \\
\hline Anxiety*** & $1.5(2.9)$ & $0-18 / 42$ & $17.19(9.87)$ & $0-38 / 42$ \\
\hline Stress*** & $4.95(6.327)$ & $0-34 / 42$ & $22.14(9.79)$ & $0-42 / 42$ \\
\hline \multicolumn{5}{|l|}{ BIS-11 } \\
\hline Total $* * *$ & $51.85(10.16)$ & $32-81 / 120$ & $64.03(11.73)$ & $39-99 / 120$ \\
\hline Attentional $* * *$ & $12.21(3.51)$ & $8-24 / 32$ & $17.75(3.73)$ & $8-26 / 32$ \\
\hline Motor*** & $18.63(3.55)$ & $11-32 / 44$ & $23.30(6.05)$ & $13-37 / 44$ \\
\hline Non-planning** & $21.01(5.80)$ & $11-38 / 44$ & 22.99 (4.89) & $12-39 / 44$ \\
\hline $\mathrm{CRT}^{* * *}$ & $2.33(1.00)$ & $0-3 / 3$ & $1.33(1.18)$ & $0-3 / 3$ \\
\hline $\mathrm{BNT}^{* * *}$ & $1.78(1.33)$ & $0-4 / 4$ & $1.02(1.14)$ & $0-4 / 4$ \\
\hline
\end{tabular}

Note: Low-OC participants $(n=165)$; High-OC participants $(n=138) ; M$ : mean; $S D$ : standard deviation; The OCI-R scores are taken from the second administration of the scale when screened participants returned for the main study. *Mean difference between low OC and high OC groups significant at $p<0.05$; $* *$ Mean difference between low OC and high OC groups significant at $p<0.01$. *** Mean difference between low OC and high OC groups significant at $p<0.001$. was re-administered in this study. Given inevitable variation in participants' time-1 versus time-2 OCI-R scores, the authors simply required participants' scores to remain above/ below the clinical cut-off score of 21 at both time points. This would allow the authors to minimize participant exclusions while still ensuring sufficiently different mean OCI-R scores for each group (see Table 1). Fifteen participants had OCI-R scores that were markedly discrepant between the two administrations and were excluded. Three others were excluded for failing an attention check, and one because their MTurk ID could not be verified.

Hence, a total of $N=303$ participants (140 female; mean age: 37.6 years) remained in the dataset. Those who were in the lower part of the distribution in the OCI-R screening and who remained below the clinical cut off score of 21 in the second OCI-R administration were categorized as low OC $(\mathrm{N}=165)$. Those who were in the upper part of the distribution in the OCI-R screening and remained above the cut off score in the second OCI-R administration were categorized as high OC $(\mathrm{N}=138)$. Low and high OC participants were randomly allocated to either the Lottery or Virus scenario (see Experimental Materials below). Participants were compensated US\$3.00-\$4.80, depending on their scenario and bonuses earnt.

\section{Experimental Materials}

Black Swan Decision Tasks The one-risk and two-risk decision tasks were adapted from Perfors and van Dam (2018) (see Fig. 1). Each task required participants to make a choice between two options with different probabilities of gains and losses, which were quantified as points. In the one-risk task, the choice was between a safer option with a moderate probability of a small points gain and no chance of a loss, and a risky option with a moderate probability of a higher points gain and a low probability that all accumulated points would be lost. In the two-risk task, the choice was between a safer option with a moderate probability of a small points gain and a very small probability of losing all points, and a more risky option with a moderate probability of a higher points gain but a low probability that all accumulated points would be lost. As shown in the Figure, the black swan outcome was always very rare (probabilities ranging between 0.0001-0.001). In each task, the safer option always had the higher expected utility based on the product of the probability and value of option outcomes (see OSF archive for expected utility calculations).

Two different scenarios were developed for the decision tasks - a financial "lottery" scenario and a health-related 
Fig. 1 Choice options for the One-Risk and Two-Risk tasks

\begin{tabular}{|c|c|c|}
\hline & One-Risk Task & Two-Risk Task \\
\hline $\begin{array}{l}\text { Safer } \\
\text { Option }\end{array}$ & $\begin{array}{l}1 \text { in } 2 \text { chance of gaining } 5 \\
\text { points }\end{array}$ & $\begin{array}{l}1 \text { in } 10,000 \text { chance of } \\
\text { losing all your current } \\
\text { points } \\
\text { and a } 1 \text { in } 2 \text { chance of } \\
\text { gaining } 6 \text { points }\end{array}$ \\
\hline $\begin{array}{l}\text { Riskier } \\
\text { Option }\end{array}$ & $\begin{array}{l}1 \text { in } 1000 \text { chance of } \\
\text { losing all your current } \\
\text { points } \\
\text { and a } 1 \text { in } 2 \text { chance of } \\
\text { gaining } 15 \text { points }\end{array}$ & $\begin{array}{l}15 \text { in } 10,000 \text { chance of } \\
\text { losing all your current } \\
\text { points } \\
\text { and a } 1 \text { in } 2 \text { chance of } \\
\text { gaining } 20 \text { points }\end{array}$ \\
\hline
\end{tabular}

"virus" scenario. In both scenarios, participants started the decision tasks with an endowment of 9000 points. Both scenarios had the same goal of making choices that would maximise the final points balance and used the same onerisk and two-risk decision options. The scenarios differed in the general framing of the decisions and meaning attached to points (see Appendix for scenario instructions). Lottery scenario instructions emphasised that maximising points would increase the bonus monetary payment, which was paid at the rate of US $\$ 0.20$ per 1000 points in the participant's balance at the end of the study. Virus scenario instructions asked participants to imagine that they were on an island with a virus outbreak. In order to save themselves and their family, they would need to win points to purchase a vaccine. The 9000point endowment would only confer moderate protection for them and their family. Virus protection would increase if additional points were earned and would decrease if the points balance fell below 9000 .

Obsessive-Compulsive Inventory-Revised (OCI-R) The OCI-R (Foa et al., 2002) is a well-validated self-report measure of OC symptoms. It contains six of the original seven Obsessive-Compulsive Inventory (OCI) subscales (Washing, Checking, Ordering, Obsessing, Hoarding, Neutralizing) and has been found to have moderate-high test-retest reliability and strong internal consistency. The OCI-R has also been found to have moderate convergent validity with observer ratings of OCD severity. The OCI-R was re-administered in the main study for reliability purposes. ${ }^{2}$

Depression, Anxiety, Stress Scales - Brief Version (DASS-21) The DASS-21 (Lovibond \& Lovibond, 1995) is a valid and reliable

\footnotetext{
$\overline{2}$ The DSM-5 (American Psychiatric Association, 2013) recognizes that hoarding is not a symptom of OCD and defines Hoarding Disorder as a separate diagnosis. Nevertheless, application of the OCI-R
}

screening measure of depression, anxiety, and tension/stress symptoms.

Barratt Impulsiveness Scale (BIS-11) The BIS-11 is a 30-item self-report measure of impulsivity (Stanford et al., 2009). In addition to a total score, the BIS-11 provides three subscales: Attentional Impulsivity, Motor Impulsivity, and NonPlanning Impulsivity. Attentional impulsiveness is defined as an inability to focus attention or concentrate. Motor Impulsiveness involves acting without thinking, and Non-Planning Impulsiveness involves a lack of "futuring" or forethought (Barratt, 1985).

Cognitive Reflection Test (CRT) The CRT is a three-item scale that is thought to measure one's ability to override prepotent cognitive responses in favour of correct answers requiring more deliberation (Frederick, 2005). CRT performance is also moderately correlated with general cognitive ability and numeracy (Campitelli \& Gerrans, 2014; Frederick, 2005).

Berlin Numeracy Test (BNT) The BNT is a brief, three-minute measure of statistical numeracy and risk literacy (Cokely et al., 2012). Specifically, the BNT measures the type of statistical numeracy required for evaluating information about risk. It has been found to have a high level of convergent validity with other measures of numeracy and general cognitive ability.

\section{Footnote 2 (continued)}

for screening and recent revisions of OCI-R norms (e.g., Abramovitch et al., 2020) continue to include the Hoarding subscale in the OCI-R total score. Note that excluding Hoarding scores from our calculation of OCI-R totals would not have changed the low/high OC classification of any participants. 


\section{Design and Procedure}

The decision task followed a 2 OC level (high vs. low) $\times 2$ scenario (lottery vs. virus) $\times 2$ black swan task type (one-risk vs. two-risk) factorial design, with repeated measures on the last factor. The presentation order of the one- and two-risk tasks was counterbalanced across participants.

All aspects of the study were coded in Java script and administered online. The study took approximately $30 \mathrm{~min}$ to complete. After providing informed consent and completing demographics questions, the study commenced with the administration of one of the decision tasks. In this task, participants first read the relevant instructions for their condition, and were then presented with three multiple-choice questions to check their comprehension (e.g., how many points they were starting with). If any question was answered incorrectly, they were taken back to the instructions screen until all questions were answered correctly. The decision task was then presented with the relevant decision options from Fig. 1 presented on screen. The left-right position of the riskier/safer options was randomized. Participants had unlimited time to make their decision and were given no immediate feedback about whether they had won or lost points.

They then completed the five individual differences questionnaires in fixed order (i.e., OCI-R, DASS-21, BIS11, CRT, BNT). In the final phase, participants received a brief reminder about the decision task instructions and then completed the second decision task. On completion, they were told how many points they had won/lost across both of the tasks. Participants in the lottery condition were also informed of their monetary bonus (maximum US\$1.80).

\section{Results}

\section{Demographics and Individual differences}

'Highest level of educational attainment' was classified as high school diploma, associate degree, undergraduate degree, professional degree or doctorate. Distribution across these categories did not differ for low OC and high OC groups, with an undergraduate degree being the most common educational qualification in both, $\chi^{2}(\mathrm{~N}=303)=2.25$, $p=0.69$. Other demographics and individual difference measures for the low OC and high OC groups are summarized in Table 1. Our design ensured a substantial separation between the distributions of OCI-R scores for the low and high OC groups. Notably, the mean OCI-R score for high OC participants was above the conventional clinical-cut off (cf. Foa et al., 2002). There was no difference between low and high OC groups in gender distribution (low OC: 72 females, 93 males; high OC: 68 females, 70 males), $\chi^{2}$
$(\mathrm{N}=303)=0.96, p=0.33$. The high OC group was significantly younger than the low OC group, $t(301)=4.63$, $p=0.03$. Table 1 shows that the high OC group had significantly higher scores than the low OC group on the total scale and all subscales of the DASS-21 and BIS-11. The high OC group had significantly lower levels of cognitive reflection (CRT) and risk numeracy (BNT) than the low OC group. All group differences remained robust when age was added as a covariate. All demographic, decision-making, and individual difference data can be accessed via the OSF archive.

Correlations between scores on the various individual difference measures are given in Table 2. Consistent with previous work (e.g., Crino \& Andrews, 1996), there was high co-morbidity between OCD symptomatology and each of the subscales on the DASS-21. OCD symptomatology was positively correlated with full-scale and sub-scale scores on the Barratt Impulsivity Scale, and negatively correlated with levels of cognitive reflection and numeracy.

\section{Decision-Making}

The order in which the one-risk and two-risk tasks were administered had no effect on the pattern of decisions $\left(\chi^{2}\right.$ $(\mathrm{N}=303)=1.9, p=0.59)$, so all subsequent analyses were collapsed across this factor. Figure 2 shows the proportion of participants in each condition who chose safer or riskier options on the one-risk and two-risk tasks for lottery and virus scenarios. For low OC participants, the proportion choosing the risky option increased significantly from the one-risk to the two-risk task, $\operatorname{McNemar}(1, N=165)=4.558$, $p=0.032$. This trend was significant in the virus scenario $(\operatorname{McNemar}(1, N=85)=4.050, p=0.041)$ but not the lottery scenario $(\operatorname{McNemar}(1, N=80)=0.696, p=0.405)$. The shift towards the more risky choice in the two-risk task replicates the general pattern reported by Perfors and van Dam (2018) in healthy adults. Notably we have shown that this pattern extends to decisions involving personally relevant, contamination-based health outcomes as well as financial decisions.

Across scenarios, the high OC group also showed a shift towards the riskier option from the one-risk to the tworisk task, McNemar $(1, N=165)=6.61, p=0.01$. Again, this effect was significant for the virus scenario (McNemar $(1, N=69)=6.500, p=0.009)$ but not the lottery scenario, $(\operatorname{McNemar}(1, N=69)=0.696, p=0.405)$.

Predicting Stability and Change in Decision-Making Because all participants completed both decision tasks, we could examine patterns of individual change and stability in risky choice. All participants were classified into one of four decision patterns across the one-risk and two-risk tasks: SaferSafer; Riskier-Riskier; Riskier-Safer; Safer-Riskier (see Fig. 3). The final category (shown in red) corresponds to the shift towards risk identified at a group level by Perfors and 


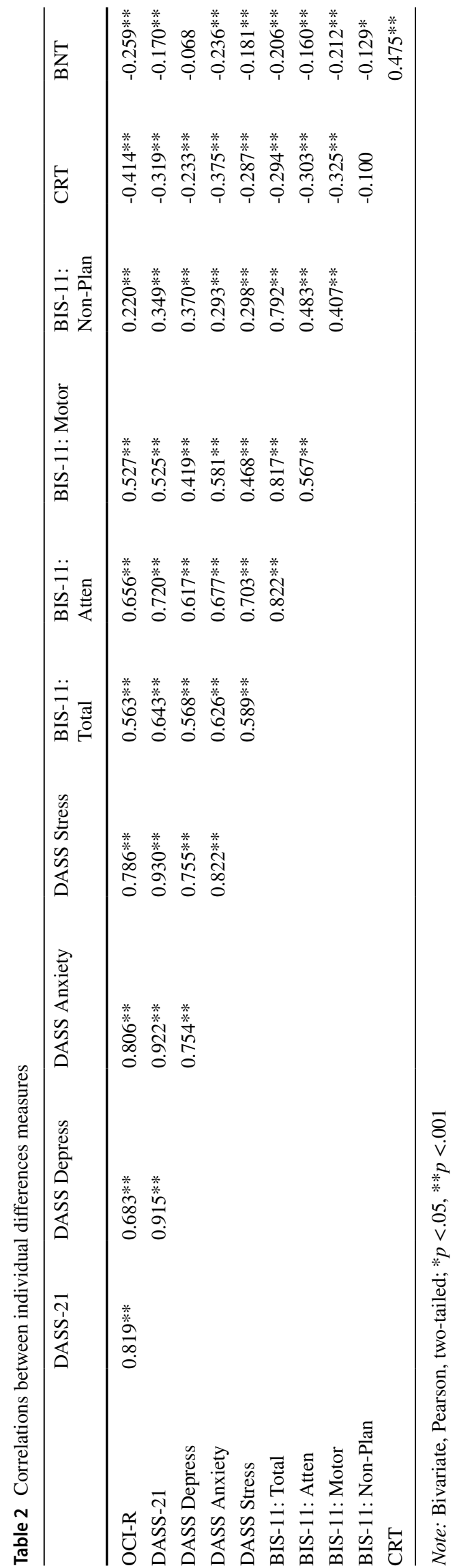

Van Dam (2018). The majority of participants (70\%) showed stable responding across the two tasks, with approximately equal proportions showing a consistent preference for safer or risky choices. When there was a shift in responding, it was more often from choice of the safer option in the one-risk task to choice of the more risky option in the two-risk task. Patterns of individual choice did not differ between low OC and high OC participants, $\chi^{2}(\mathrm{~N}=303)=3.479, p=0.324$, or between scenarios, $\chi^{2}(\mathrm{~N}=303)=5.028, p=0.170$. It also did not differ between younger (35 years and less) and older participants (36-77 years), irrespective of OC status, $\chi^{2}(\mathrm{~N}=303)=1.978, p=0.577$.

A further analysis examined whether those in the four decision pattern categories differed in their scores on the various individual difference measures (see Table 3). There were no significant differences between the four decision categories on the OCI-R total score, BIS-11 total score, BIS11 Attentional score, BIS-11 Motor score, DASS-21 total score, or BNT (all $F$ 's $<2.2$ ). However, there were differences between decision categories in scores on the BIS-11 Non-planning scale, $F(1,295)=3.292, p=0.02, \eta_{\mathrm{P}}^{2}=0.03$, and CRT, $F(1,295)=2.651, p=0.049, \eta_{\mathrm{P}}^{2}=0.03$. Table 3 indicates that those who showed the shift from the safer choice on the one-risk task to the riskier choice on the tworisk task showed elevated levels of impulsivity and lower levels of cognitive reflection compared to those in the other decision categories. These effects did not vary across scenarios, $F$ 's $<1.6$.

\section{Discussion}

This study sought to extend the existing body of research on risky decision-making in OCD. An important novel feature was the incorporation of decisions which included "black swan" options, characterized by very rare events that could lead to extremely negative outcomes. A further novel feature was the examination of decisions involving both financial outcomes and outcomes perceived as having personally relevant health outcomes (specifically, contamination-based outcomes of particular relevance to clients with OCD).

Following a previous study with healthy adults (Perfors \& Van Dam, 2018), we examined choices on a one-risk task where only one of the choice options could lead to a black swan loss, and a two-risk task where both options involved black swan outcomes, although one was more probable (by a factor of 15). Like Perfors and Van Dam (2018), we found that for the one-risk task, most individuals low on OCD symptomatology preferred the safer option where there was no possibility of a black swan outcome. Such choices are in line with normative models of decision-making like expected utility theory (cf. Buchholz \& Schymura, 2012). Many low OC individuals however, shifted to the objectively 
Fig. 2 Proportion of participants in each group choosing the Safer versus Riskier option for each task; Low OC Lottery group $(n=80)$; Low OC Virus group $(\mathrm{n}=85)$; High OC Lottery group $(n=69)$; High OC Virus group $(\mathrm{n}=69)$

\section{Low OC - Lottery}

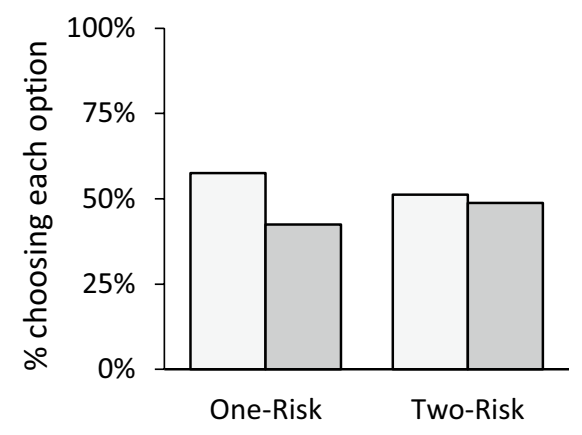

Low OC - Virus

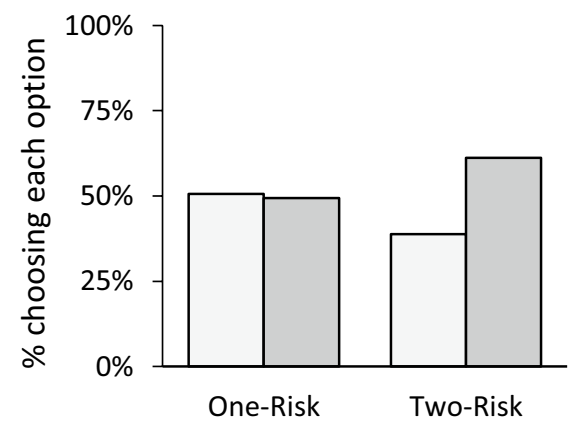

High OC - Lottery

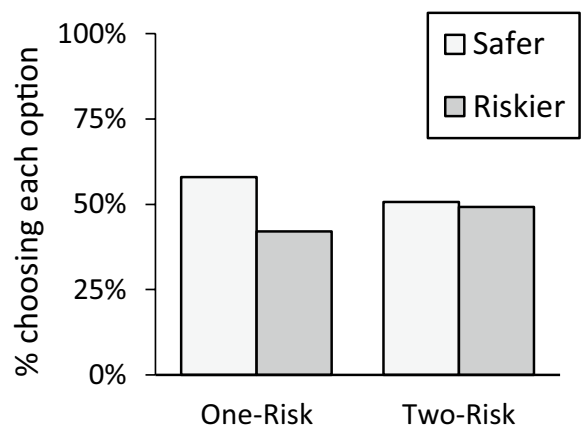

High OC - Virus

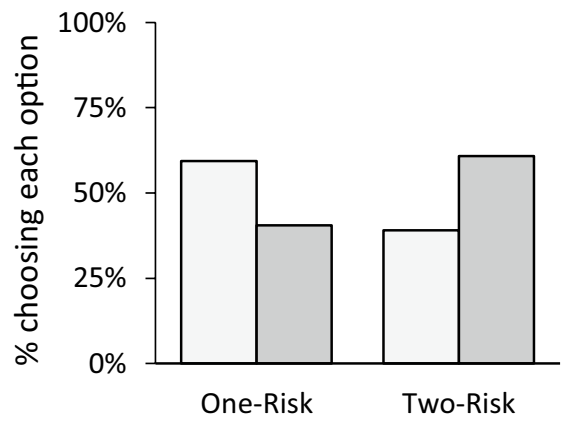

riskier option on the two-risk task, in violation of the normative prescription. Notably, those with higher levels of OCD symptomatology showed a broadly similar pattern, with a preference for the safe option on the one-risk task and a shift towards riskier choices on the two-risk task.

These patterns suggest that: a) participants approached the one-risk and two-risk tasks in qualitatively different ways and b) this was true for both high and low OC participants. When there was an option with no risk of a loss, this was generally preferred by both groups. When the decision was between options, each of which had a very low risk of loss, participants appeared to ignore the relative magnitudes of risk. Instead, they appeared to focus attention on reward probabilities, leading to increased choice of the riskier option. Notably, this shift towards more risky choice was not related to participant numeracy or general statistical knowledge as measured by the BNT.

These results show that both high and low OC decisionmakers often do not behave normatively when faced with deciding between options that both entail risk of substantial loss. In such cases, they appear to treat both options as "risky" and base their choice on other features of the options (e.g., the potential gains each option offers). This is an important result given that such decisions are likely to be much more common in the everyday environment than "risk free" options like those in the one-risk task.
A further interesting and novel finding was that, for both high and low OC individuals, the shift towards risky choices was especially evident when outcomes were OCD-relevant, involving issues of health and responsibility. This reinforces the view that the patterns of non-normative decision-making that we observed in the two-risk task are likely to be found in a range of decision contexts relevant to OCD.

Although we used decision tasks that are arguably more similar to the types of everyday decisions that are challenging for people with OCD, we found broadly similar patterns of responding in those with many or few OCrelated symptoms. This could suggest that there is continuity between decision processes in those with and without OCD (cf. Pushkarskaya, et al., 2015, 2017; Starcke, et al., 2010). That is, both groups show a preference for riskless options where possible, but face difficulty in deciding between options when both entail a substantial negative risk.

An alternative interpretation is that a transdiagnostic factor such as impulsivity that is correlated with obsessive-compulsive symptoms is a better predictor of problematic decision-making in black swan situations. We found some evidence for this, with those who shifted to the risky option in the two-risk task showing higher scores on the non-planning scale of the BIS-11 and lower scores on the CRT. The BIS-11 non-planning scale is thought to measure 
Fig. 3 Percentage of Low OC and High OC participants exhibiting four decision patterns in lottery and virus scenarios
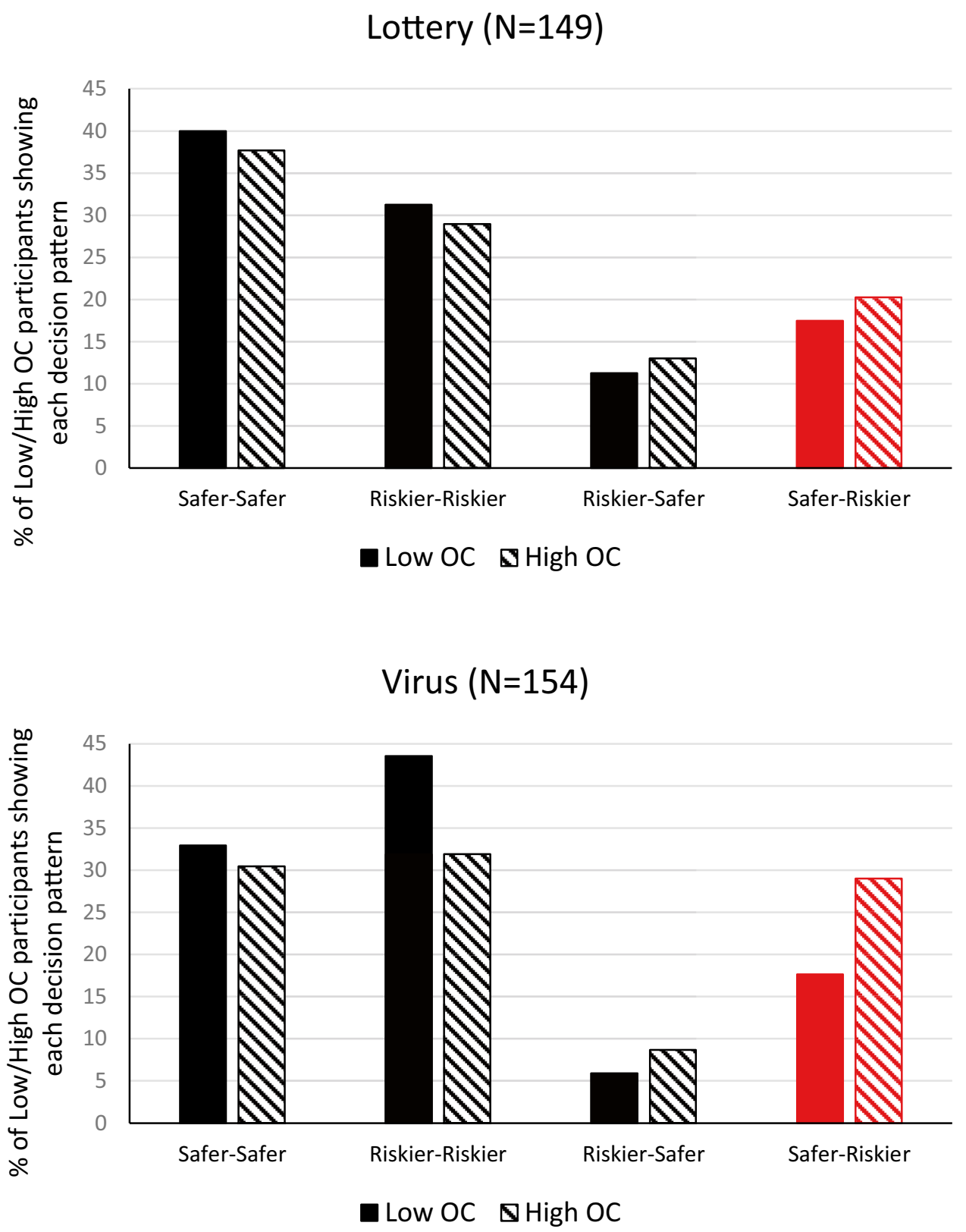

the tendency to avoid thinking about difficult problems or a lack of forethought (Stanford et al., 2009). Low CRT scores may reflect a tendency to respond intuitively and without deliberation (Frederick, 2005). Because this relationship was found on only a single BIS-subscale it should be treated with caution. However, the view that impulsivity may be a factor mediating problems in decision-making across a range of psychological disorders is supported by other work (Chamberlain et al., 2018; Gillan et al., 2016; Grassi, et al., 2015). A clearer picture of the role of impulsivity in black swan decision-making may emerge from future work that includes other clinical groups known to show high levels of impulsivity, such as those with Attention-Deficit/Hyperactivity Disorder (ADHD) (Ruscio et al., 2010).
There are two additional ways that the current results on black-swan decision-making in OCD could be progressed. First, rather than just relying on performance on "one-shot" decision tasks, participants varying in OCD symptoms could be presented with a larger suite of decisions varying in risk probabilities and outcomes. This would yield a larger body of individual response data which may provide a more sensitive test of shifts towards risky responding in patient groups. It also would allow for mathematical modelling of the relative influence of different decision components such as the probability and magnitude of decision outcomes (e.g., Aranovich, et al., 2017).

Second, the current paradigm could be modified by replacing specific probabilities of black swan outcomes with less 
Table 3 Individual differences profile for the four categories of individual decision-making

\begin{tabular}{lllll}
\hline & $\begin{array}{l}\text { Safer-Safer } \\
M(S D)\end{array}$ & $\begin{array}{l}\text { Riskier-Riskier } \\
M(S D)\end{array}$ & $\begin{array}{l}\text { Riskier-Safer } \\
M(S D)\end{array}$ & $\begin{array}{l}\text { Safer-Riskier } \\
M(S D)\end{array}$ \\
\hline OCI-R & $21.00(18.99)$ & $19.40(18.66)$ & $23.69(19.08)$ & $23.56(17.39)$ \\
DASS-21 & $29.31(30.03)$ & $31.90(33.93)$ & $31.93(30.52)$ & $40.38(33.34)$ \\
BIS-11 & & & \\
Total & $56.08(11.32)$ & $57.40(13.36)$ & $55.24(11.09)$ & $60.62(13.03)$ \\
Attentional & $14.46(4.37)$ & $14.56(4.78)$ & $15.07(4.68)$ & $15.33(4.41)$ \\
Motor & $20.15(5.12)$ & $21.26(5.63)$ & $19.72(5.35)$ & $21.43(5.31)$ \\
Non-planning* & $21.48(5.07)$ & $21.59(5.75)$ & $20.45(3.88)$ & $23.86(5.96)$ \\
CRT* & $1.94(1.16)$ & $2.08(1.16)$ & $1.62(1.24)$ & $1.56(1.24)$ \\
BNT & $1.50(1.30)$ & $1.56(1.33)$ & $1.34(1.50)$ & $1.14(1.15)$ \\
\hline
\end{tabular}

Note: Safer-Safer $(n=107)$; Riskier-Riskier $(n=104)$; Riskier-Safer $(n=29)$, Safer-Riskier $(n=63) ; M$ : mean; $S D$ : standard deviation. *Mean difference between decision styles significant at $p=0.05$. precise descriptions (e.g., "the chance of losing all points is between 1 in 1000 and 1 in 10,000" or even "the chance of losing all points is extremely low"). This converts the task into an ambiguous rather than a risky decision-making task (Camerer \& Weber, 1992). As well as providing a better simulation of real-world decisions, where the precise risk probabilities are rarely known, this change is motivated by recent evidence that suggests that those with OCD may have particular difficulty in decisions involving ambiguous outcome probabilities (Pushkarskaya, et al., 2015, 2017).

Although we did not find evidence of substantial differences in black swan decision-making between those with high and low OC symptoms, the current work does reinforce an important message for the treatment of OCD. When the decision options offered an opportunity to completely avoid a black swan risk, the majority of those with high OC symptoms did so. In the real world however, clinicians may emphasise that riskless decision options are rare. Moreover, a quest to find such risk-free options can lead to paralysis or extreme avoidance with respect to behaviour and decision-making (e.g., avoiding public bathrooms completely). Hence, interventions like graduated exposure to situations that encourage clients to tolerate low levels of risk of negative outcomes, are necessary to help those with OCD reduce their anxiety and make more adaptive decisions (cf. Tolin \& Steketee, 2007).

\section{Conclusions}

To better simulate the types of decisions that are problematic in OCD we used a novel black swan decision paradigm where there was a very low risk of a highly negative outcome. We found evidence that both high OC and low OC individuals responded in different ways to a task where there was an option to avoid risk, and a task where they had to choose between options that both had a low risk of a black swan outcome. Many participants, particularly those who were high in impulsivity, shifted to a more risky option in the latter case. These trends were especially pronounced for contamination-based, health-related decisions. We see these findings as an important first step in uncovering the mechanisms that underlie disordered decision-making in black swan scenarios in OCD and related disorders.

\section{Appendix}

\section{Lottery Scenario Instructions}

This experiment involves making choices between different options. No matter how you do here you'll be guaranteed to receive the $\$ 3.00$ that you signed up for. However, if your choices pay off you may earn a bonus on top of that! Here's how it will work. You are allocated 9000 points to begin with. Every 1000 points is worth 20 cents of bonus payment: thus, with these initial points if the task was ending now you would be paid $\$ 4.80$ (the $\$ 3.00$ guaranteed rate plus $\$ 1.80$ bonus $[9 \times 20$ cents $=\$ 1.80]$ ).

In this task you will be asked to make bets about different possibilities with different payoffs and losses. At the end we will use the computer to simulate a game that follows the choices you made. Your bonus will be based on the outcome of these games.

Click 'Next' to answer a few questions showing that you understood these instructions, and then you'll finally be ready to do the task!

\section{Virus Scenario Instructions}

This part of this experiment involves making choices between different options. Here's how it will work. You are about to read a fictitious scenario. Please try your 
best to imagine the events as if they were really happening to you. Take a moment to imagine that you and your family are on an island. On this island, a highly infectious virus has broken out. The virus starts with fever, fatigue and sore throat. As it progresses, it causes vomiting, diarrhea, rashes, bleeding, and eventually leads to death. You notice that more and more people are getting infected each day. A vaccine can be purchased to protect you and your family from the virus. This vaccine can be purchased with points, with the level of protection provided by the vaccine increasing with points. You currently have 9000 points, which gives you and your family a moderate level of protection from the virus. But note that this level of protection may not be enough to ensure that your family is safe. You will be asked to make bets about different possibilities with different points payoffs and losses. At the end we will use the computer to simulate games that follow the choices you made. Your total points - and your level of protection from the virus - will be based on the outcomes of those games. Click 'Next' to answer a few questions showing that you understood these instructions, and then you'll finally be ready to do the task!

Acknowledgements We would like to thank Peggy Wei for her assistance in data collection and Harrison Kusevskis-Hayes for his assistance in coding the online tasks.

Funding This research was supported by an Australian Research Council Discovery Project Grant DP190101224 to BKH and an Australian Research Council Discovery Project Grant DP180103600 to AP.

\section{Declarations}

Conflicts of Interest/Competing Interests The authors have no conflicts of interest to declare that are relevant to the content of this article.

Ethics Approval This research was approved by the University of New South Wales Australia Human Research Ethics Advisory Panel (3126).

Consent Informed consent was obtained from all individual participants included in the study.

\section{References}

Abramovitch, A., Shaham, N., Levin, L., Bar-Hen, M., \& Schweiger, A. (2015). Response inhibition in a subclinical obsessive-compulsive sample. Journal of Behavior Therapy and Experimental Psychiatry, 46, 66-71. https://doi.org/10.1016/j.jbtep.2014.09.001

Abramovitch, A., Abramowitz, J. S., Riemann, B. C., \& McKay, D. (2020). Severity benchmarks and contemporary clinical norms for the Obsessive-Compulsive Inventory-Revised (OCI-R). Journal of Obsessive-Compulsive and Related Disorders, 27, 100557.

Abramowitz, J. S., Franklin, M. E., Zoellner, L. A., \& Dibernardo, C. L. (2002). Treatment compliance and outcome in obsessive-compulsive disorder. Behavior Modification, 26(4), 447-463.
Abramowitz, J. S., Fabricant, L. E., Taylor, S., Deacon, B. J., McKay, D., \& Storch, E. A. (2014). The relevance of analogue studies for understanding obsessions and compulsions. Clinical Psychology Review, 34(3), 206-217. https://doi.org/10.1016/j.cpr. 2014.01.0

Admon, R., Bleich-Cohen, M., Weizmant, R., Poyurovsky, M., Faragian, S., \& Hendler, T. (2012). Functional and structural neural indices of risk aversion in obsessive-compulsive disorder (OCD). Psychiatry Research: Neuroimaging, 203(2-3), 207-213.

American Psychiatric Association. (2013). Diagnostic and Statistical Manual of Mental Disorders (5th ed.). (DSM-5). American Psychiatric Association.

Aranovich, G. J., Cavagnaro, D. R., Pitt, M. A., Myung, J. I., \& Mathews, C. A. (2017). A model-based analysis of decision making under risk in obsessive-compulsive and hoarding disorders. Journal of Psychiatric Research, 90, 126-132. https://doi.org/10. 1016/j.jpsychires.2017.02.017

Barratt, E. S. (1985). Impulsiveness subtraits: Arousal and information processing. Motivation, Emotion and Personality, 99, 137-146.

Buchholz, W., \& Schymura, M. (2012). Expected utility theory and the tyranny of catastrophic risks. Ecological Economics, 77, 234-239.

Camerer, C., \& Weber, M. (1992). Recent developments in modeling preferences: Uncertainty and ambiguity. Journal of Risk and Uncertainty, 5(4), 325-370.

Campitelli, G., \& Gerrans, P. (2014). Does the cognitive reflection test measure cognitive reflection? A mathematical modeling approach. Memory \& Cognition, 42(3), 434-447.

Chamberlain, S. R., Stochl, J., Redden, S. A., \& Grant, J. E. (2018). Latent traits of impulsivity and compulsivity: Toward dimensional psychiatry. Psychological Medicine, 48(5), 810-821.

Cicolini, T., \& Rees, C. S. (2003). Measuring Risk-Taking In ObsessiveCompulsive Disorder: An Extension Of The Everyday Risk Inventory With An Australian Sample. Behavioural and Cognitive Psychotherapy, 31(3), 247.

Cokely, E. T., Galesic, M., Schulz, E., Ghazal, S., \& Garcia-Retamero, R. (2012). Measuring Risk Literacy: The Berlin Numeracy Test. Judgment and Decision Making, 7(1), 25-47. https://doi.org/10. 1146/annurev.psych.49.1.447

Crino, R. D., \& Andrews, G. (1996). Obsessive-compulsive disorder and axis I comorbidity. Journal of Anxiety Disorders, 10(1), $37-46$.

Fineberg, N. A., Chamberlain, S. R., Goudriaan, A. E., Stein, D. J., Vanderschuren, L. J. M. J., Gillan, C. M., ... Potenza, M. N. (2014). New developments in human neurocognition: clinical, genetic, and brain imaging correlates of impulsivity and compulsivity. CNS Spectrums, 19(1), 69-89. https://doi.org/10.1017/S1092852913000801

Foa, E. B., Huppert, J. D., Leiberg, S., Langner, R., Kichic, R., Hajcak, G., \& Salkovskis, P. M. (2002). The Obsessive-Complusive Inventory: Development and validation of a short version. Psychological Assessment, 14(4), 485-495. https://doi.org/10.1037//1040-3590. 14.4.485

Frederick, S. (2005). Cognitive Reflection and Decision Making. Journal of Economic Perspectives, 19(4), 25-42. https://doi.org/10. $1257 / 089533005775196732$

Fontenelle, L. F., Oostermeijer, S., Harrison, B. J., Pantelis, C., \& Yücel, M. (2011). Obsessive-compulsive disorder, impulse control disorders and drug addiction. Drugs, 71(7), 827-840.

Gillan, C. M., Kosinski, M., Whelan, R., Phelps, E. A., \& Daw, N. D. (2016). Characterizing a psychiatric symptom dimension related to deficits in goal-directed control. Elife, 5, e11305.

Grassi, G., Pallanti, S., Righi, L., Figee, M., Mantione, M., Denys, D., ... Stratta, P. (2015). Think twice: Impulsivity and decision making in obsessive-compulsive disorder. Journal of Behavioral Addictions, 4(4), 263-272. https://doi.org/10.1556/2006.4.2015. 039 
Hertwig, R., \& Erev, I. (2009). The description-experience gap in risky choice. Trends in Cognitive Sciences, 13(12), 517-523.

Koran, L. M., \& Simpson, H. B. (2013). Guideline watch (March 2013): practice guideline for the treatment of patients with obsessive-compulsive disorder. American Psychiatric Association.

Lovibond, P. F., \& Lovibond, S. H. (1995). The structure of negative emotional states: Comparison of the Depression Anxiety Stress Scales (DASS) with the Beck Depression and Anxiety Inventories. Behaviour Research and Therapy, 33(3), 335-343.

Olatunji, B. O., Davis, M. L., Powers, M. B., \& Smits, J. A. (2013). Cognitive-behavioral therapy for obsessive-compulsive disorder: A meta-analysis of treatment outcome and moderators. Journal of Psychiatric Research, 47(1), 33-41.

Perfors, A., \& Van Dam, N. T. (2018). Human decision making in black swan situations. In T. T. Rogers, M. Rau, X. Zhu, \& C. W. Kalish (Eds.), Proceedings of the 40th Annual Conference of the Cognitive Science Society (pp. 870-876). Cognitive Science Society.

Pushkarskaya, H., Tolin, D., Ruderman, L., Henick, D., Kelly, J. M., Pittenger, C., \& Levy, I. (2017). Value-based decision making under uncertainty in hoarding and obsessive-compulsive disorders. Psychiatry Research, 258, 305-315.

Pushkarskaya, H., Tolin, D., Ruderman, L., Kirshenbaum, A., Kelly, J. M. L., Pittenger, C., \& Levy, I. (2015). Decision-making under uncertainty in obsessive-compulsive disorder. Journal of Psychiatric Research, 69, 166-173. https://doi.org/10.1016/j.jpsychires. 2015.08.011

Ruscio, A. M., Stein, D. J., Chiu, W. T., \& Kessler, R. C. (2010). The epidemiology of obsessive-compulsive disorder in the National Comorbidity Survey Replication. Molecular Psychiatry, 15(1), 53.

Salkovskis, P. M., Wroe, A. L., Gledhill, A., Morrison, N., Forrester, E., Richards, C., ... Thorpe, S. (2000). Responsibility attitudes and interpretations are characteristic of obsessive compulsive disorder. Behaviour Research and Therapy, 38(4), 347-372. https://doi.org/10.1016/S0005-7967(99)00071-6

Schulze, D., Kathmann, N., \& Reuter, B. (2018). Getting it just right: A reevaluation of OCD symptom dimensions integrating traditional and Bayesian approaches. Journal of Anxiety Disorders, 56, 63-73.

Sip, K. E., Muratore, A. F., \& Stern, E. R. (2016). Effects of context on risk taking and decision times in obsessive-compulsive disorder. Journal of Psychiatric Research, 75, 82-90.

Sohn, S. Y., Kang, J. I., Namkoong, K., \& Kim, S. J. (2014). Multidimensional measures of impulsivity in obsessive-compulsive disorder: Cannot wait and stop. PLoS One, 9(11). https://doi.org/ 10.1371/journal.pone.0111739

Stanford, M. S., Mathias, C. W., Dougherty, D. M., Lake, S. L., Anderson, N. E., \& Patton, J. H. (2009). Fifty years of the Barratt Impulsiveness Scale: An update and review. Personality and Individual Differences, 47(5), 385-395. https://doi.org/10.1016/j.paid.2009.04.008

Starcke, K., Tuschen-Caffier, B., Markowitsch, H. J., \& Brand, M. (2010). Dissociation of decisions in ambiguous and risky situations in obsessive-compulsive disorder. Psychiatry Research, 175(1-2), $114-120$

Taleb, N. N. (2007). The black swan: The impact of the highly improbable (Vol. 2). Random house.

Tolin, D. F., \& Steketee, G. (2007). General Issues in Psychological Treatment for Obsessive-Compulsive Disorder. In Psychological treatment of obsessive-compulsive disorder: Fundamentals and beyond. (pp. 31-59). American Psychological Association.

von Neumann, J., \& Morgenstern, O. (1944). Theory of games and economic behavior. John Wiley.

Publisher's Note Springer Nature remains neutral with regard to jurisdictional claims in published maps and institutional affiliations. 